\title{
Cálculo dos vetores de posição e velocidade dos satélites GLONASS a partir das efemérides transmitidas e aspectos relacionados à sua integração com o GPS
}

\author{
Thiago STATELLA ${ }^{1}$, Claudinei R. AGUIAR², João F. G. MONICO³ \& José R. NOGUEIRA \\ ${ }^{1}$ Instituto Federal de Educação, Ciência e Tecnologia de Mato Grosso. Rua Professora Zulmira Canavarros, 93, Centro, CEP 78005-200, \\ Cuiabá, MT, Brasil. E-mail: thiago.statella@cba.ifmt.edu.br. \\ ${ }^{2}$ Universidade Tecnológica Federal de Pato Branco. Via do Conhecimento, km 1, CEP 85503-390, Pato Branco, PR, Brasil. E-mail: rodrigues. \\ aguiar@gmail.com. \\ ${ }^{3}$ Universidade Estadual Paulista, Faculdade de Ciências e Tecnologia. Rua Roberto Simonsen, 305, CEP 19060-900, Presidente Prudente, \\ SP, Brasil. E-mail: galera@fct.unesp.br, jrobnog@gmail.com.
}

Recebido em 07/2012. Aceito para publicação em 06/2013.

Versão online publicada em xx/xx/20xx (www.pesquisasemgeociencias.ufrgs.br)

\begin{abstract}
Resumo - Este trabalho apresenta detalhes sobre o cálculo dos vetores de posição e velocidade dos satélites GLONASS a partir de suas efemérides transmitidas, utilizando o método de integração de Runge-Kutta de quarta ordem e a compatibilização das efemérides GLONASS (GLobal Orbiting NAvigation Sattelite System) com o sistema de tempo GPS (Global Positioning System). Também é feita uma análise da compatibilização entre os sistemas de tempo GLONASS e GPS. Para análise da qualidade do método de integração, as coordenadas extrapoladas foram comparadas às coordenadas transmitidas. A média das discrepâncias foi de 1,33 $\mathrm{m}$, com desvio padrão de $\pm 0,83 \mathrm{~m}$. Para demonstrar a compatibilização entre os sistemas (de tempo) GPS e GLONASS, as coordenadas GLONASS calculadas a partir das efemérides transmitidas foram comparadas com as coordenadas precisas geradas pelo IGS no tempo GPS. A discrepância média foi de 6,53 m, menor que a precisão (divulgada) das efemérides transmitidas para o GLONASS. Em seguida, foi feita a compatibilização entre os sistemas (de tempo) GPS e GLONASS ao se calcular as coordenadas GLONASS a partir das efemérides transmitidas, no tempo GPS.
\end{abstract}

Palavras-chave: GLONASS, GPS, Órbitas, Integração Numérica, Runge-Kutta.

\begin{abstract}
CALCULATING POSITION AND VELOCITY VECTORS OF GLONASS SATELLITES FROM BROADCAST EPHEMERIS AND ASPECTS RELATED TO ITS INTEGRATION WITH GPS. This paper presents details on the estimation of position and velocity vectors for GLONASS satellites from their broadcasted ephemeris using forth-order Runge-Kutta integration method and deals with the compatibility between GLONASS ephemeris and GPS time system. Besides, we analyze the compatibility between GLONASS and GPS time systems. In order to assess the quality of the integration method the coordinates calculated were compared to the broadcasted ones. The mean discrepancy was $1.33 \mathrm{~m}$ with a standard deviation of $\pm 0.83 \mathrm{~m}$. Next, we showed how to make GPS and GLONASS (time) systems compatible by calculating GLONASS coordinates from broadcasted ephemeris in GPS time. The results were compared to the IGS coordinates. The mean discrepancy was $6.53 \mathrm{~m}$ which agrees with the GLONASS broadcasted ephemeris precision.
\end{abstract}

Keywords: GLONASS, GPS, orbits, numeric integration, Runge-Kutta.

\section{Introdução}

O sistema de posicionamento global desenvolvido pela antiga União Soviética e hoje sob responsabilidade da Rússia, denominado GLONASS (GLobal Orbiting NAvigation Sattelite System), apresenta diversas similaridades ao sistema GPS (Global Positioning System), desenvolvido pelo Departamento de Defesa dos Estados Unidos. A utilização conjunta das duas constelações proporciona um maior recobrimento da superfície terrestre e aumenta o número de satélites visíveis em determinados horários e em determinadas regiões, garantindo maior confiabilidade dos resultados, uma vez que, além de uma quantidade maior de observações disponíveis, soluções independentes poderão ser comparadas.
Ambos os sistemas foram desenvolvidos para proporcionar posicionamento 3-D, velocidade 3-D e informações de tempo em quaisquer condições climáticas, em níveis local, regional e global; quando totalmente operacionais, disponibilizam ao menos 24 satélites (embora a disposição dos satélites seja diferente nos dois sistemas); posições são calculadas com base em medidas de distância entre receptores e satélites. Cada um dos sistemas é composto pelos segmentos espacial, de controle e de usuários (Cheng, 1998; Monico, 2008; ICD, 2008). As principais diferenças começam com o referencial adotado em cada sistema. Enquanto o GPS utiliza o sistema WGS84 (World Geodetic System 1984), o GLONASS utiliza o PZ90 (Parametry Zemli 1990). Os dois sistemas possuem idealizações semelhantes: a origem é o centro de massa da Terra; o eixo 
Z aponta para o Pólo Terrestre Convencional (do inglês Conventional Terrestrial Pole - CTP), de acordo com a recomendação do IERS (International Earth Rotation and Reference Systems Service); o eixo X aponta para a interseção do equador terrestre no meridiano origem, conforme estabelecido pelo IERS; e o eixo Y completa o sistema dextrógiro. Porém, pequenas diferenças entre suas materializações e entre os elipsóides adotados em cada um deles precisam ser levadas em conta. Como exemplo, parâmetros de transformação entre WGS84 e PZ90 podem ser encontrados em Cheng (1998), Habrich (1999), Balzov et al. (1999), Boucher \& Altamimi (2001), Zinoviev (2005) e Gaglione et al. (2009).

Além dos sistemas de referência espaciais, GPS e GLONASS adotam sistemas de tempo distintos e conectados a realizações diferentes de UTC (Coordinated Universal Time). 0 sistema de tempo GLONASS $\left(t_{G L}\right)$ (gerado na central sincronizadora de tempo GLONASS) apresenta um offset constante de $3 \mathrm{~h}$ (correspondentes à diferença de tempo entre Moscou e Greenwich) em relação ao UTC(SU) (UTC Russo (SU) medido pelo Main Metrological Center of Russian Time and Frequency Service). 0 tempo GLONASS, portanto, considera os leap seconds (saltos de segundos entre o TU (Tempo Universal) e o TAI (Tempo Atômico Internacional)), ao contrário do tempo GPS (Lewandowski \& Arias, 2011). Também existe uma discrepância da ordem de poucos microssegundos entre o tempo GLONASS e UTC(SU), decorrente de oscilações no tempo medido pelos relógios que realizam os dois referenciais. Esta diferença $\left(\tau_{c}\right)$ é informada nas efemérides transmitidas e, como exemplo, era de $\sim 0,136 \times 10^{-6}$ s em 07/09/2009. 0 tempo UTC(SU) pode então ser calculado a partir da relação $t_{U T C(S U)}=t_{G L}+\tau_{c}-3 h .0$ sistema de tempo GPS $\left(t_{G P S}\right)$ é uma escala de tempo contínua iniciada em 6 de janeiro de 1980 e difere do UTC(USNO) (UTC medido pelo US Naval Observatory) pela quantidade de leap secon$d s$ (decorridos desde 1980), bem como por pequenas discrepâncias, da ordem de nanossegundos, entre o tempo medido pelos relógios que materializam o $t_{G P S} \mathrm{e}$ o $t_{\text {UTC(USNO) }}($ Rossbach, 2000; ICD, 2008). Como exemplo, em $07 / 09 / 2009$ havia $15 \mathrm{~s}$ de salto entre as duas escalas de tempo.

Para obter a posição instantânea da antena de um receptor o usuário deve ter acesso às posições e ao sistema de tempo dos satélites em tempo real. Estas informações estão contidas nas efemérides transmitidas. De uma maneira geral as efemérides são produzidas com base em modelos que consideram as forças de atração gravitacional da Terra, Sol e Lua, dentre outros. Aqui aparece uma diferença marcante entre os sistemas GPS e GLONASS: no primeiro, as órbitas são transmitidas na forma de elementos keplerianos e, no segundo, em coordenadas cartesianas, acompanhadas das respectivas velocidades e da aceleração causada pela atração luni-solar, em intervalos de $30 \mathrm{~min}$. As coordenadas do satélite para uma época $t_{i}$ diferente da época de referência $t_{b}, \operatorname{com}\left|t_{b}-t_{i}\right|<15$ min, são obtidas via integração numérica das equações diferenciais do modelo de força dos satélites. A integração precisa ser realizada em um referencial inercial (ou quase inercial), mas, por causa do curto período de integração, a precessão, nutação e o movimento do pólo podem ser negligenciados e apenas o efeito da rotação da Terra é considerado (ICD, 2008). 0 método de integração recomendado pelo GLONASS ICD (GLONASS Interface Control Document) é o Runge-Kutta de quarta ordem.

Stewart \& Tsakiri (1998) abordaram a questão da determinação dos vetores de posição e velocidade de satélites GLONASS a partir das efemérides transmitidas. Os autores fizeram uma breve discussão sobre o modelo de força orbital e deram detalhes do processo de integração numérica. Alguns experimentos sobre a influência da variação no passo de integração nos resultados foram feitos. Os autores também mostraram a diminuição da precisão das coordenadas obtidas ao se extrapolar o intervalo de integração recomendado pelo ICD (2008). Em ambos os casos as coordenadas assumidas como sendo referência e utilizadas na comparação foram aquelas transmitidas na mensagem de navegação dos satélites. Zinoviev (2005) discutiu aspectos relativos à utilização conjunta do sistema GPS e GLONASS. 0 autor compara resultados de integração numérica para cálculo das efemérides GLONASS utilizando os métodos de Runge-Kutta (quarta ordem), Fehlberg (quinta ordem) e Shanks (sétima ordem) e mostra que eles são equivalentes para um passo de integração de até 5 segundos. No trabalho também foi abordada a transformação de coordenadas entre os sistemas WGS84 e PZ90. Lin et al. (2009) discutiram a utilização da função ODE45, implementada no software Matlab, para a integração das equações diferenciais de movimento dos satélites. Os autores utilizaram as equações simplificadas (ICD, 2008), porém, consideraram uma função linear para variação da atração gravitacional luni-solar no intervalo de integração de \pm 15 min, obtendo resultados mais precisos. Hu et al. (2009) também se dedicaram ao problema de calcular posições e velocidades dos satélites GLONASS com base nas efemérides transmitidas. Os autores utilizaram os métodos de interpolação de Lagrange \& Chebyshev e compararam com os resultados obtidos pela integração com o método de Runge-Kutta de quarta ordem. Para determinar a qualidade dos métodos utilizaram as coordenadas transmitidas em instantes posteriores $\left(t_{b}+30 \mathrm{~min}\right)$ ou anteriores $\left(t_{b}-30 \mathrm{~min}\right)$ às da época considerada.

Porém, nenhum dos trabalhos citados explora em detalhes a necessidade de se fazer a compatibilização entre os referenciais de tempo para que a integração entre GPS e GLONASS possa ser feita. Este trabalho visa apresentar detalhes sobre o cálculo dos vetores de posição e velocidade dos satélites GLONASS a partir de suas efemérides transmitidas e a compatibilização destas com o sistema de tempo GPS. Para a integração numérica foi utilizado o método Runge-Kutta de quar- 
ta ordem, recomendado por ICD (2008). Para análise da qualidade do método de integração as coordenadas extrapoladas foram comparadas às coordenadas transmitidas (no instante $t_{b}+30 \mathrm{~min}$, em que $t_{b}$ foi o instante tomado como origem da integração). Também foi feita uma comparação entre as coordenadas extrapoladas no intervalo de 15 min para frente e para trás (no tempo) entre duas atualizações consecutivas das coordenadas transmitidas. Finalmente, para análise do resultado da compatibilização entre os sistemas de tempo GLONASS e GPS, as coordenadas calculadas a partir das efemérides transmitidas (originalmente no tempo GLONASS) foram comparadas com as coordenadas precisas, no tempo GPS, geradas pelo IGS (International GNSS Service).

\section{Métodos}

A seguir são apresentados o modelo de força perturbadora orbital, que descreve o comportamento dos satélites GLONASS e o método de integração de quarta ordem de Runge-Kutta, utilizado para calcular as efemérides dos satélites.

\subsection{Modelo de força perturbadora orbital}

As efemérides GLONASS contêm o vetor de posição, velocidade e aceleração luni-solar para seus satélites no instante $t_{b}$. Estes dados são atualizados a cada 30 minutos e são válidos para um intervalo $\left|t_{b}-t_{i}\right|<$ 15 min. Para obter as coordenadas dos satélites num instante $t_{i}$ as equações que governam seus movimentos precisam ser integradas. A principal força atuante sobre os satélites é a atração gravitacional da Terra, expressa como (Stewart \& Tsakiri, 1998):

$$
V=\frac{\mu}{r}+U
$$

Em que $V$ é o potencial gravitacional total, $\mu=G M$ é a constante gravitacional da Terra, $r=\left(x^{2}+y^{2}+z^{2}\right)^{1 / 2}$ é a distância do satélite ao centro da Terra (módulo do raio vetor) e $U$ é a componente não esférica do potencial gravitacional da Terra.

De acordo com Heiskenan \& Moritz (1967), a expansão de $U$ em harmônicos esféricos resulta em:

$U=\frac{\mu}{r} \sum_{n=2}^{\infty} \sum_{m=0}^{n}\left(\frac{a_{E}}{r}\right)^{n} P_{n m}(\cos \theta)\left(c_{n m} \cos m \lambda+s_{n m} \sin m \lambda\right)$

Em que $a_{E}$ é o raio equatorial terrestre, $(r, \lambda, \theta)$ são coordenadas polares no sistema terrestre (raio vetor, longitude e colatitude), ( $n, m) \operatorname{com} n \geq m$ são o grau e a ordem da expansão do harmônico esférico, $\left(P_{n m}(\cos i)\right)$ são as funções associadas de Legendre e $\left(C_{n m} S_{n m}\right)$ são os coeficientes dos harmônicos esféricos.

Os harmônicos zonais (independentes da latitude) são muito mais significantes para as órbitas dos satélites do que os demais, portanto a equação 2 , com grau $n$ qualquer e $m=0$, pode ser simplificada e reescrita da seguinte maneira:

$$
U=\frac{\mu}{a_{E}} \sum_{n=2}^{\infty}\left(\frac{a_{E}}{r}\right)^{n+1} c_{n 0} P_{n 0}(\cos \theta)
$$

Outras forças, além da gravidade da Terra, também perturbam o movimento dos satélites. Algumas delas e suas respectivas magnitudes são apresentadas na tabela 1.

Tabela 1. Magnitude de forças atuantes sobre o movimento dos satélites. Fonte: adaptada de Spilker (1996). *Potencial gravitacional da Terra.

\begin{tabular}{cc}
\hline Força perturbadora & $\begin{array}{c}\text { Magnitude depois de 1h } \\
\text { (m) }\end{array}$ \\
\hline $\begin{array}{c}\text { Segundo harmônico zonal }\left(c_{20}\right) \\
\text { *Harmônico zonal de grau } 2 \text { do } \\
\text { geopotencial }(n>2)\end{array}$ & 300 \\
Demais harmônicos & 0,6 \\
Gravidade lunar & 0,06 \\
Gravidade solar & 40 \\
Pressão da radiação solar & 20 \\
\hline
\end{tabular}

Pela tabela 1 percebe-se que as principais forças são causadas pelo segundo harmônico zonal e pela atração luni-solar. Negligenciando-se as demais fontes de perturbação e substituindo-se $P_{20}(\cos \theta)=3 / 2 \cos ^{2} \theta-1 / 2$ (polinômio de Legendre para grau 2) a equação (3) pode ser reescrita como (Stewart \& Tsakiri, 1998):

$$
U=\frac{\mu a_{E}^{2}}{r^{3}} c_{20}\left(\frac{3}{2} \cos ^{2} \theta-\frac{1}{2}\right)
$$

Portanto, o potencial gravitacional total pode ser escrito como:

$$
V=\frac{\mu}{r}+\frac{\mu a_{E}^{2}}{r^{3}} c_{20}\left(\frac{3}{2} \cos ^{2} \theta-\frac{1}{2}\right)
$$

E o vetor de aceleração $\ddot{\vec{X}}$ dos satélites, em coordenadas cartesianas, causada pela atração gravitacional, é dado pelo gradiente do potencial gravitacional (Stewart \& Tsakiri, 1998):

$$
\ddot{\vec{X}}=\nabla V
$$

As componentes do vetor de aceleração são calculadas como:

$$
\ddot{\vec{X}}_{i}=\frac{d V}{d X_{i}}=\frac{\partial V}{\partial r} \frac{\partial r}{\partial X_{i}}+\frac{\partial V}{\partial \lambda} \frac{\partial \lambda}{\partial X_{i}}+\frac{\partial V}{\partial \theta} \frac{\partial \theta}{\partial X_{i}}
$$


E após o cálculo das derivadas parciais obtém-se:

$$
\begin{aligned}
& \ddot{x}=-\frac{\mu}{r^{3}} x+\frac{3}{2} c_{20} \frac{\mu a_{E}^{2}}{r^{5}} x\left(1-5 \frac{z^{2}}{r^{2}}\right)+\ddot{x}_{L S} \\
& \ddot{y}=-\frac{\mu}{r^{3}} y+\frac{3}{2} c_{20} \frac{\mu a_{E}^{2}}{r^{5}} y\left(1-5 \frac{z^{2}}{r^{2}}\right)+\ddot{y}_{L S} \\
& \ddot{z}=-\frac{\mu}{r^{3}} z+\frac{3}{2} c_{20} \frac{\mu a_{E}^{2}}{r^{5}} z\left(3-5 \frac{z^{2}}{r^{2}}\right)+\ddot{z}_{L S}
\end{aligned}
$$

Em que $x, y, z$ dá a posição do satélite e $\ddot{x}_{L S^{\prime}} \ddot{\mathrm{Y}}_{L S^{\prime}} \mathrm{e}$ $\ddot{z}_{L S}$ são as componentes de atração gravitacional (fornecidos nas efemérides) luni-solar, assumidas constantes dentro do intervalo de integração $( \pm 15 \mathrm{~min})$.

A equação 8 é aplicável apenas a sistemas inerciais. Para se obter as coordenadas do satélite num sistema terrestre é necessário considerar a rotação da Terra (os demais efeitos, como movimento do pólo, precessão e nutação podem ser negligenciados dentro do intervalo de integração). Portanto, as equações que governam o movimento dos satélites podem ser definidas da maneira como segue:

$$
\begin{gathered}
V_{x}=\dot{x} \\
V_{y}=\dot{y} \\
V_{z}=\dot{z} \\
\ddot{x}=-\frac{\mu}{r^{3}} x+\frac{3}{2} c_{20} \frac{\mu a_{E}^{2}}{r^{5}} x\left(1-5 \frac{z^{2}}{r^{2}}\right)+\ddot{x}_{L S}+w_{E}^{2} x+2 w_{E} \dot{y} \\
\ddot{y}=-\frac{\mu}{r^{3}} y+\frac{3}{2} c_{20} \frac{\mu a_{E}^{2}}{r^{5}} y\left(1-5 \frac{z^{2}}{r^{2}}\right)+\ddot{y}_{L S}+w_{E}^{2} y+2 w_{E} \dot{x} \\
\ddot{z}=-\frac{\mu}{r^{3}} z+\frac{3}{2} c_{20} \frac{\mu a_{E}^{2}}{r^{5}} z\left(3-5 \frac{z^{2}}{r^{2}}\right)+\ddot{z}_{L S}
\end{gathered}
$$

Tabela 2. Acurácia das efemérides transmitidas GLONASS. Fonte: ICD (2008).

\begin{tabular}{c|c|c|c|c}
\hline \multirow{2}{*}{ Componente } & \multicolumn{4}{|c}{ Erro médio quadrático } \\
\cline { 2 - 5 } & \multicolumn{2}{|c}{ Coordenadas (m) } & \multicolumn{2}{c}{ Velocidades $\left(\mathrm{cm} \times \mathrm{s}^{-1}\right)$} \\
\hline SV & GLONASS & GLONASS-M & GLONASS & GLONASS-M \\
\hline Transversal & 20 & 7 & 0,05 & 0,03 \\
\hline Normal & 10 & 7 & 0,1 & 0,03 \\
\hline
\end{tabular}

Tabela 3. Parâmetros do sistema de referência PZ90. Fonte: ICD (2008).

\begin{tabular}{ll}
\hline Parâmetros & Valores \\
\hline Taxa de rotação da Terra $\left(w_{E}\right)$ & $7,292115 \times 10^{-5} \mathrm{rad} \times \mathrm{s}^{-1}$ \\
Constante gravitacional $(\mu)$ & $398.600,4418 \times 10^{9} \mathrm{~m}^{3} \times \mathrm{s}^{-2}$ \\
Raio equatorial $\left(a_{E}\right)$ & $6.378 .136 \mathrm{~m}$ \\
${ }^{*}$ Harmônico zonal de grau 2 do geopotencial $\left(c_{20}\right)$ & $1082625,75 \times 10^{-9}$ \\
\hline
\end{tabular}




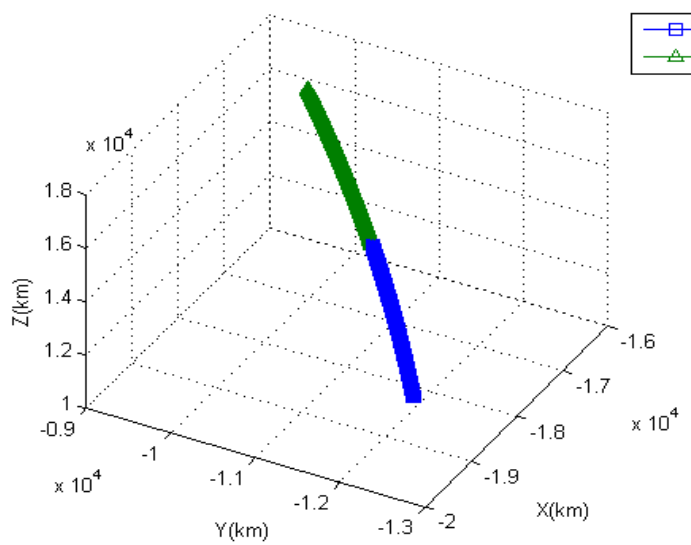

Figura 1. Trajetória do satélite 11 no intervalo 00:00:00 h a 00:30:00 h do dia 09/07/2009.

A figura 2 mostra a velocidade do satélite 11 no intervalo de tempo considerado.

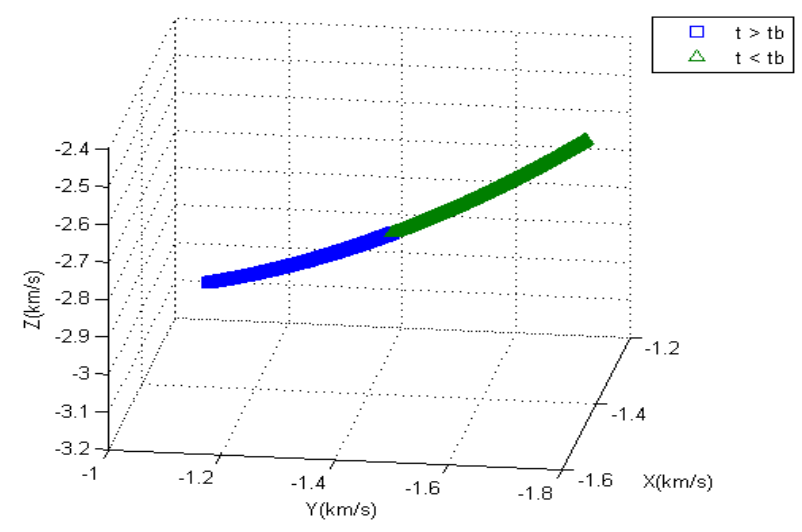

Figura 2. Velocidade do satélite 11 no intervalo 00:00:00 h a 00:30:00 h do dia 09/07/2009.

A figura 3 mostra o cálculo das coordenadas do mesmo satélite 11 , desta vez num período de $24 \mathrm{~h}$.

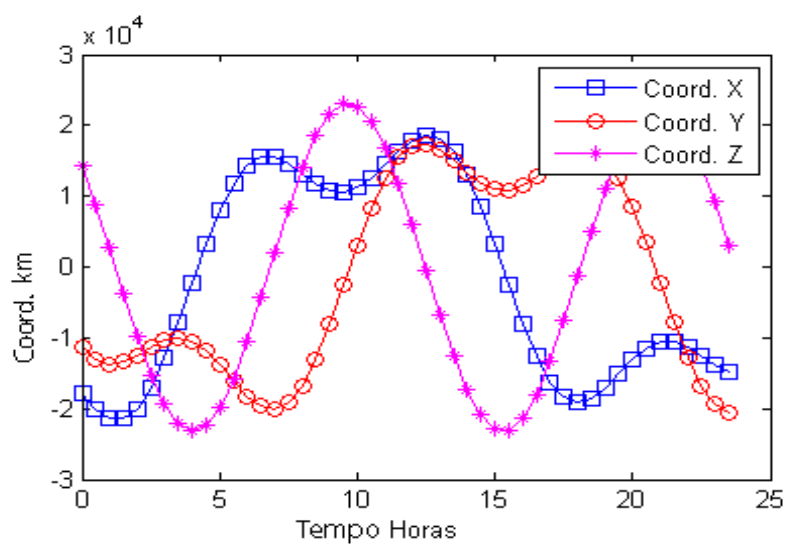

Figura 3. Coordenadas do satélite 11 no intervalo de 24 h do dia 09/07/2009.

Em seguida, com a finalidade de mostrar a degradação da precisão das coordenadas integradas ao se extrapolar o intervalo recomendado de $\pm 15 \mathrm{~min}$, foram calculadas as coordenadas do satélite 11 para um intervalo de $2 \mathrm{~h}$ (com passo de integração de $1 \mathrm{~s}$ ), a contar do instante $t_{b}=00: 15: 00 \mathrm{~h}$. As coordenadas calculadas nos instantes $t=00: 45: 00 \mathrm{~h}, 01: 15: 00 \mathrm{~h}, 01: 45: 00 \mathrm{~h}$ e 02:15:00 h foram comparadas com as coordenadas transmitidas nas efemérides. As discrepâncias são apresentadas na figura 4. A figura 5 mostra as discrepâncias entre as velocidades estimadas com a integração e as transmitidas nas efemérides. Pode-se observar que os erros nas posições e nas velocidades não são maiores que $2 \mathrm{~m}$ e $0,25 \mathrm{~cm} \times \mathrm{s}^{-1}$, respectivamente, no período de $\sim 30$ min (2.000 s). Porém, suas precisões se degradam rapidamente após este intervalo de tempo, chegando a $\sim 40 \mathrm{~m}$ (erro máximo em posição, na coordenada X) e

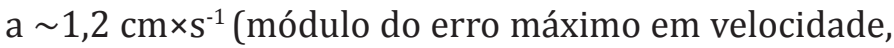
na componente $\mathrm{Y}$ ) ao final de $2 \mathrm{~h}(7.200 \mathrm{~s})$, para o caso estudado. Estes resultados coincidem com os obtidos por Stewart \& Tsakiri (1998) e Lin et al. (2009). Deve-se ressaltar que parte destes erros advém das simplificações feitas ao modelo de força orbital que governa o movimento dos satélites, bem como do fato de que a aceleração luni-solar foi considerada constante no intervalo de integração (conforme recomendações feitas por ICD (2008)).

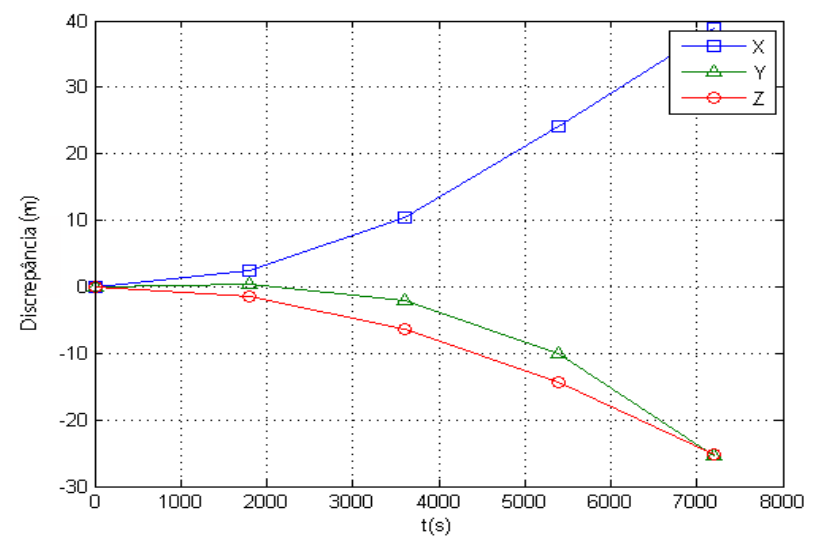

Figura 4. Discrepâncias entre coordenadas integradas e transmitidas para o satélite $11 \mathrm{em}$ um intervalo de $2 \mathrm{~h}$.

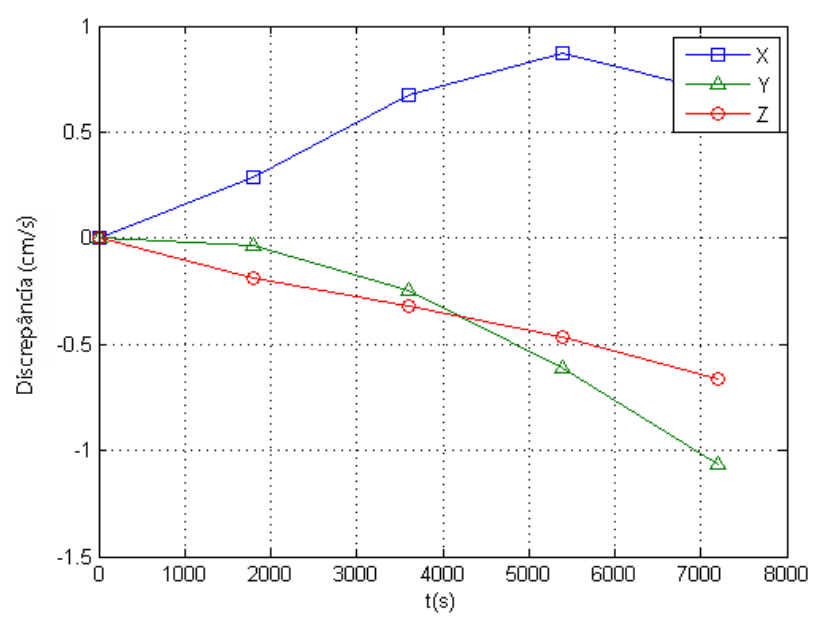

Figura 5. Discrepâncias entre velocidades integradas e transmitidas para o satélite 11 em um intervalo de $2 \mathrm{~h}$. 
0 erro da integração numérica das coordenadas extrapoladas no limite do intervalo $( \pm 15 \mathrm{~min})$ recomendado pelo GLONASS ICD (ICD, 2008) pode ser avaliada ao se comparar as coordenadas extrapoladas dos satélites para frente e para trás no tempo. Para os satélites $2,3,7,8,10,11,13,14,15,17,18,19,20,21$, 23 e 24, em operação na data de referência, foram calculadas as discrepâncias entre suas coordenadas no instante 00:30:00 h, obtidas da integração numérica a partir dos instantes de referência $t_{b}=00: 15: 00 \mathrm{~h}$ (posições integradas para frente) e $t_{b}=00: 45: 00 \mathrm{~h}$ (posições integradas para trás). A figura 6 mostra os resultados. A média das discrepâncias foi de 1,33 m, com desvio padrão de $\pm 0,83 \mathrm{~m}$. 0 valor máximo foi de $3,35 \mathrm{~m}$ (satélite 13) e o valor mínimo foi de $0,36 \mathrm{~m}$ (satélite 11 ). Nos experimentos de Stewart \& Tsakiri (1998), para um passo de integração de $1 \mathrm{~s}$ as discrepâncias foram menores que $2 \mathrm{~m}$. Neste trabalho, apenas dois satélites apresentaram erro superior $2 \mathrm{~m}$ : os satélites 13 e 23 . Isso pode ser explicado pela ausência de informação sobre a aceleração daqueles satélites nas efemérides transmitidas. Ao serem desconsiderados os satélites 13 e 23, a média das discrepâncias é de 1,08 m, com desvio padrão de $\pm 0,51 \mathrm{~m}$ e com discrepância máxima de 1,87 m. É preciso ter em mente que estes erros são fortemente influenciados pela precisão das efemérides transmitidas e pela validade do modelo de força para o período de integração de \pm 15 min.

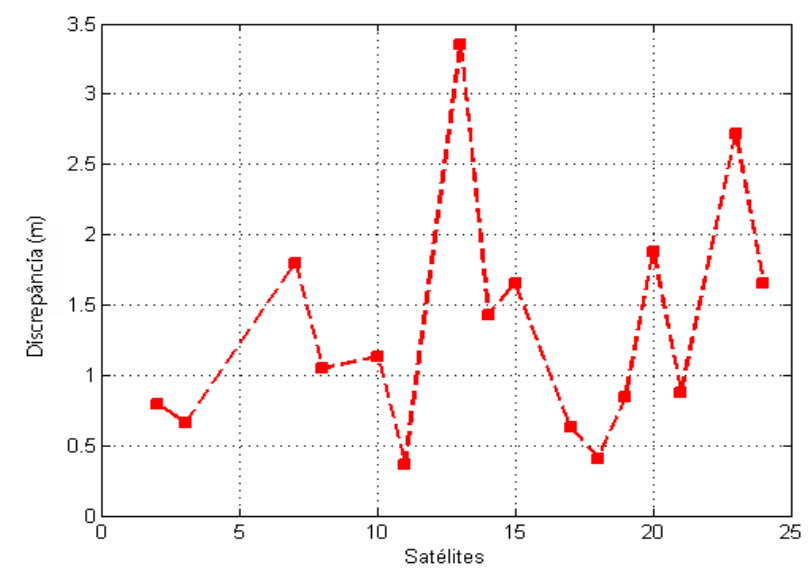

Figura 6. Discrepâncias entre as posições dos satélites quando são comparadas as coordenadas integradas para frente e para trás (no tempo).

Até o momento os cálculos foram feitos no sistema de tempo GLONASS $\left(t_{C L}\right)$. Mas, para que a utilização conjunta dos sistemas GLONASS e GPS seja possível, é preciso que as posições dos satélites da constelação russa sejam calculadas no mesmo sistema de tempo, por exemplo, GPS $\left(t_{G P S}\right)$ (tomando-se este sistema como referência para a adequação). Uma escala de tempo pode ser convertida na outra pela seguinte relação:

$$
t_{b}^{G P S}=t_{b}^{G L}-3 h-\left(T A I-t_{G L}\right)+\left(T A I-t_{G P S}\right)
$$

Em que $t_{b}^{G P S}$ e $t_{b}^{G L}$ são, respectivamente, o tempo
GPS e o tempo GLONASS no instante $b$.

As diferenças entre o Tempo Atômico Internacional e as escalas de tempo GPS e GLONASS são dadas por:

$$
\begin{aligned}
& \left(T A I-t_{G L}\right)=34 s+C_{1} \\
& \left(T A I-t_{G P S}\right)=19 s+C_{0}
\end{aligned}
$$

Os valores de $C_{1}$ e $C_{0}$ são rotineiramente publicados na circular T do BIPM (Bureau International des Poids et Mesures). Para o dia 09/07/2009, segundo a circular T 259 de $07 / 08 / 2009$, os valores são $C_{1}=66,8$ ns (nano-segundos) \pm 12 ns e $C_{0}=-9,4$ ns $\pm 2,8$ ns. Portanto, a equação (13) para a data de referência fica:

$$
t_{b}^{G P S}=t_{b}^{G L}-15,0000000762 s
$$

As posições dos satélites $2,3,7,8,10,11,13,14$, $15,17,18,19,20,21,23$ e 24 foram calculadas no tempo GPS para o instante 00:30:00 h, a partir das efemérides transmitidas no instante 00:15:00 h e comparadas com as posições calculadas pelo IGS (também no tempo GPS) às 00:30:00 h. As discrepâncias são mostradas na Figura 7. A média das discrepâncias foi de $6,53 \mathrm{~m}$ com desvio padrão de $3,47 \mathrm{~m}$. A discrepância máxima foi 14,65 m (para o satélite 23) e a mínima foi 2,58 m (para o satélite 7). Mais uma vez, a grande discrepância encontrada para o satélite 23 pode ter sido causada pela ausência de informação sobre sua aceleração nas efemérides transmitidas. Em média, os valores obtidos concordam com as precisões das efemérides transmitidas $(7 \mathrm{~m})$, conforme a tabela 2 .

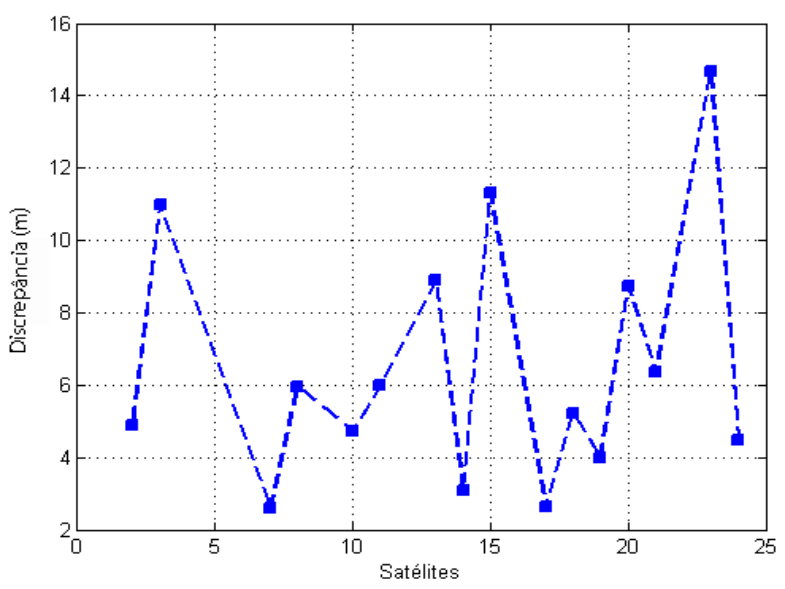

Figura 7. Discrepâncias entre posições calculadas a partir das efemérides transmitidas e as posições calculadas pelo IGS.

\section{Conclusão}

Este trabalho visou apresentar detalhes sobre o cálculo dos vetores de posição e velocidade dos satélites GLONASS a partir de suas efemérides transmitidas e a compatibilização destas com o sistema de tempo GPS. Há alguns trabalhos (Stewart \& Tsakiri, 1998; Zinoviev, 2005; Hu et al., 2009) que tratam do cálculo das coordenadas GLONASS porém a abordagem do assunto 
não é exaustiva. 0 mesmo acontece com a compatibilização entre os sistemas de tempo GLONASS e GPS. Este trabalho contribui com maiores detalhes sobre o tema. A integração numérica foi feita pelo método Runge-Kutta de quarta ordem, recomendado por ICD (2008). Para análise da qualidade do método de integração as coordenadas extrapoladas foram comparadas às coordenadas transmitidas (no instante $t_{b}+30 \mathrm{~min}$, em que $t_{b}$ foi o instante tomado como origem da integração). A média das discrepâncias foi de $1,33 \mathrm{~m}$, com desvio padrão de $\pm 0,83 \mathrm{~m}$, resultados que concordam com os obtidos por Stewart \& Tsakiri (1998). Apenas dois satélites apresentaram erro superior $2 \mathrm{~m}$ : os satélites 13 e 23. Isso pode ser explicado pela ausência de informação sobre a aceleração daqueles satélites nas efemérides transmitidas.

Para demonstrar a compatibilização entre os sistemas (de tempo) GPS e GLONASS, as coordenadas GLONASS calculadas (no tempo GPS) a partir das efemérides transmitidas (originalmente no tempo GLONASS) foram comparadas com as coordenadas precisas geradas pelo IGS no tempo GPS. A discrepância média $(6,53 \mathrm{~m})$ concorda com a precisão das efemérides transmitidas para o GLONASS.

\section{Referências}

Balzov, Y.A., Galazin, V.F., Kaplan, B.L., Maksimov, V.G. \& Rogozin, V. P. 1999. Propagating PZ90 to WGS84 transformation parameters. GPS Solutions, 1(3): 13 - 16.

Boucher, C. \& Altamimi, Z. 2001. ITRS, PZ-90 and WGS84: current realizations and the related transformation parameters. Journal of Geodesy, 11(75): 613 - 619.

Cheng, C. 1998. Calculations for positioning with the global Navigation Satellite System. Ohio, 96 p. Dissertação de Mestrado, College of Engineering, Ohio University.

Gaglione, S., Angrisano, A., Pugliano, G., Robustelli, U., Santa- maria, R. \& Vultaggio, M. 2011. A stochastic sigma model for GLONASS satellite pseudorange. Applied Geomatics, 3(1): 49 - 57.

Habrich, H. 1999. Geodetic Applications of the Global Navigation Satellite System (GLONASS) and of GLONASS/GPS Combinations. Berna, 124 p. Dissertação de Mestrado, Faculdade de Ciências de Berna.

Heiskenan, W.A. \& Moritz, H. 1967. Physical Geodesy. São Francisco, W. H. Freeman \& Co Ltda, 364 p.

Hu, L., Yuan, C. \& Fang, L. 2009. Extrapolation and fitting algorithms for GLONASS satellite orbit. In: INTERNATIONAL SYMPOSIUM ON INTELLIGENT INFORMATION TECHNOLOGY APPLICATION, 3, 2009, Nanchang, China. Anais..., Nanchang, p. 282 - 285.

ICD. Interface Control Document. 2008. Moscou 2008, Ed 5.1, Russian Institute of Space Device Engineering. Disponível em <http://rniikp.ru/en/pages/about/publ/ICD_GLONASS_eng.pdf>, acesso em out. 2010.

Lin, Y., Guo, H. \& Yu, M. 2009. A comparison for GLONASS satellite coordinate calculation. In: INTERNATIONAL CONFERENCE ON INFORMATION ENGINEERING AND COMPUTER SCIENCE - ICIECS, 2009, Anais..., Wuhan, China, p. 1 - 4 .

Monico, J.F.G. 2008. Posicionamento pelo GNSS: descrição, fundamentos e aplicações, 2.ed. São Paulo, Ed. UNESP, 480 p.

Rossbach, U. 2000. Positioning and Navigation Using the Russian Satellite System GLONASS. Munique, 159 p. Tese de Doutorado, Universidade de Munique, Faculdade de Engenharia Civil e Agrimensura.

Spilker, J.J. 1996. GPS Navigation Data. In: Parkinson, B.W. \& Spilker, J.J. Global Positioning System: Theory and Applications I, v. 163 of Progress in Astronautics and Aeronautics, Washington, American Institute of Aeronautics and Astronautics, p. 121-176.

Stewart, M. \& Tsakiri, M. 1998. GLONASS broadcast orbit computation. GPS Solutions, 2(2): 16-27.

Zinoviev, A. E. 2005. Using GLONASS in combined GNSS receivers: current status. In: ION GNSS INTERNATIONAL TECHNICAL MEETING OF THE SATELLITE DIVISION, 18, Anais ..., Long Beach, Estados Unidos , p. 1046 - 1057.

Manuscrito 499

Editores: Marcelo Matsvoka e Cesar L. Schultz. 